understanding of hospice care is lacking in trainee pharmacists due to minimal hospice training. The Experiential-LearningTrajectory helped improve the trainees' confidence in understanding a pharmacists' contribution to hospice care. Further placements and evaluation using higher levels of Moore's Evaluation Framework is planned.

\section{P-177 EXPLORATION OF ATTENDANCE ACROSS 28 ECHO PROJECT ECHO NETWORKS IN THE CONTEXT OF EVERYDAY PRACTICE}

${ }^{1} J a n e t$ Diffin, ${ }^{1}$ Tracey McTernaghan, ${ }^{2}$ Aine McMullan, ${ }^{2}$ Martin Hayes, ${ }^{1}$ Max Watson, ${ }^{1}$ Christopher Jenkins. ${ }^{1}$ Hospice UK, Belfast, UK; ${ }^{2}$ Health and Social Care Board, Belfast, UK

\subsection{6/spcare-2021-Hospice.193}

Introduction Many studies that have included reports of attendance at ECHO sessions have focused on networks that have purposively selected participants to attend. This data may not provide an accurate reflection of participation rates within the constraints of everyday practice.

Aim The aim of this study was to explore attendance patterns across ECHO networks attended by both medical and social care professionals within the confines of normal working practice, and identify the reasons for any observed variations.

Methods Network activity data were collected from 28 ECHO networks to include the number of ECHO sessions delivered within each network and the number attended by each participant. The number of 'low' (attended 1-32\% of sessions), 'medium' (attended 34-67\% of sessions) and 'high attenders' (attended $\geq 68 \%$ of sessions) for each network were calculated. Data were examined and explanations for observed attendance patterns were extracted.

Results The majority of networks delivered nine ECHO sessions held monthly. 1588 participants attended at least once across all 28 networks. Variations in the proportion of low, medium and high attenders were observed across the networks. In $20 / 28$ networks $\geq 50 \%$ of participants were 'low' attenders. Reasons for low attendance included competing workplace demands and staff shortages. However, interview data indicated that for several networks with a high proportion of 'low attenders' participation in the network was at service rather than individual level. Impacts at a service level were still identified through qualitative data collection. Networks with a larger proportion of 'medium' or 'high' attenders were characterised by strong leadership and/or a link to wider service developments.

Conclusion It needs to be considered what outcomes are realistic to assess based on levels of attendance and number of ECHO sessions delivered. Qualitative data collection is recommended to unpick reasons for observed attendance patterns and identify any unexpected outcomes.

\section{P-178 USING THE CONSOLIDATED FRAMEWORK FOR IMPLEMENTATION RESEARCH (CFIR) TO EXPLORE THE USE OF PROJECT ECHO}

${ }^{1}$ Janet Diffin, ${ }^{1}$ Tracey McTernaghan, ${ }^{2}$ Aine McMullan, ${ }^{2}$ Martin Hayes, ${ }^{1}$ Max Watson, ${ }^{1}$ Christopher Jenkins. ${ }^{1}$ Hospice UK, Belfast, UK; ${ }^{2}$ Health and Social Care Board, Belfast, UK
Introduction The Health and Social Care Board identified Project ECHO as a methodology to implement change. The aim was to develop the knowledge and skills of health and social care professionals, transform service delivery, and ultimately improve patient care. Up to 38 ECHO networks across a variety of settings (e.g. palliative care, ophthalmology) were established between 2018-2020.

Aim The aim of this study was to use the Consolidated Framework for Implementation Research (CFIR) to guide assessment of the outcomes and impacts, and explore the factors which influence success across different contexts.

Methods Qualitative study; key stakeholders including high and low attenders, and hub members were purposively invited to participate in a semi-structured interview; interview schedules guided by the CFIR method utilised for data analysis.

Results 28 stakeholders participated. Network purpose varied across contexts, (i) provision of education and support to isolated practitioners; (ii) support practical implementation of strategies/guidelines across sites, (iii) support cross sector working, (iv) build capacity of GPs to manage patients on waiting lists, (v) transform and provide ongoing governance of a new service. Impacts on participants included increased knowledge of conditions and symptoms, increased confidence to treat patients, and increased capacity to manage patients in primary care. Improvements in relationships between primary and secondary care, and across sectors were also reported e.g. improved understanding of regional strategies, service initiatives and referral pathways. Impacts at service level were reported.

Conclusion Project ECHO is an ideal methodology for not only delivering education and support to health and social care professionals, but also as a mechanism for delivering service change. CFIR is a useful framework for identifying preconditions which should be met before a network is operational to ensure success and sustainability.

\section{P-179 A JOINT AGENCY APPROACH TO PROMOTING WELLBEING AND RESILIENCE IN CARE HOME STAFF}

Jane Hazeldine. Mountbatten, Isle of Wight, UK

10.1136/spcare-2021-Hospice. 195

A regular multi-agency meeting 'The Ethical Task Force Group' was created in response to COVID-19 pandemic. It was recognised that staff, managers and owners of care homes were experiencing unique challenges during the pandemic. The task force comprised the Isle of Wight council, the Isle of Wight-Care Partnership and Mountbatten Hospice. The group met weekly to discuss concerns and issues relevant to all parties in order to respond to the needs of care home staff.

One main concern raised was the emotional impact the pandemic was having on care home staff. Many homes lost multiple residents in a short space of time. Often they had known their residents for many years and described their bereavement as the same as losing a family member. They were unable to acknowledge the death in the usual way and therefore were left coping with the ongoing stress of caring for their residents, whilst unable to access their usual support networks and process their bereavement. Both owners and managers of care homes were keen to access strategies and 